\title{
Expression of E-cadherin in Colorectal Cancer and Its Association with Morphological Features
}

\author{
Shahina Parambattu Hydru' ${ }^{1}$ Nisha M. Das ${ }^{2}$ \\ 1, 2 Department of Pathology, Government Medical College, KUHS (Kerala University of Health Sciences) \\ Thrissur, Kerala, India.
}

\section{ABSTRACT}

\section{BACKGROUND}

Early diagnosis of colorectal cancer is associated with a good prognosis and uncovering the biological mechanisms underlying pathogenesis and progression of this cancer form the recent focus of research. E-cadherin is one of the strong intercellular adhesion molecules in epithelial cells and one of the best characterized markers of epithelial-mesenchymal transition. The objectives of this study were to assess the pattern of expression of E-cadherin in colorectal cancer in patients from a medical college in Kerala, India and also determine the association between the expression of E-cadherin and different morphological parameters as well as the grade and stage of the tumour.

\section{METHODS}

All specimens of histologically diagnosed colorectal cancers received in the department were included in the study. Immunohistochemical staining of formalinfixed paraffin-embedded tissue was done. The intensity of E-cadherin staining was scored and analyzed for different tissues. Frequency tables of different variables were analyzed using the chi-square test. The significance of the correlation between different variables was assessed using Fischer's exact test. P-value $<0.05$ was considered statistically significant.

\section{RESULTS}

A total of 43 specimens of histologically diagnosed colorectal cancers were analyzed for the study. The mean age of the participants was 58 years. Majority of the tumours were $<5 \mathrm{~cm}$ in size. Both homogenous $(55.8 \%)$ and heterogeneous staining (44.2\%) were noted in the E-cadherin staining pattern among the participants. In patients with low E-cadherin index, 12 of them showed a heterogeneous pattern of expression. E-cadherin expression was not statistically associated with tumour differentiation in the patients.

\section{CONCLUSIONS}

There is a significant difference in E-cadherin expression between low and highgrade tumours of colorectal cancer. High-grade tumours showed a more heterogeneous staining pattern correlating with increased invasiveness of the tumour. The heterogeneous pattern of staining in E-cadherin among adjacent cells of tumour results from reduced expression of the protein in the tumour.
Corresponding Author:

Dr. Nisha M. Das,

Sooryakanthi, M. R. Vasu Road,

CRA-22, Ayyanthole, Thrissur-680003,

Kerala, India.

E-mail: drnishamohandas75@gmail.com

DOI: $10.14260 /$ jemds/2022/31

How to Cite This Article:

Hydru SP, Das NM. Expression of Ecadherin in colorectal cancer and its association with morphological features. J Evolution Med Dent Sci 2022;11(01):163168, DOI: $10.14260 / \mathrm{jemds} / 2022 / 31$

Submission 10-01-2022,

Peer Review 23-01-2022,

Acceptance 25-01-2022,

Published 29-01-2022.

Copyright (c) 2022 Shahina Parambattu Hydru et al. This is an open access article distributed under Creative Commons Attribution License [Attribution 4.0 International (CC BY 4.0)]

\section{KEY WORDS}

E cadherin, Colorectal Cancer, Cancer Marker. 


\section{BACKGROUND}

Colorectal cancer is one of the most common types of malignancies and one of the leading causes of mortality and morbidity worldwide. ${ }^{1}$ About $50 \%$ of the patients with this cancer develop liver metastases during their lifetime. ${ }^{2}$ Metastatic stages of colorectal cancer are associated with an increased rate of mortality and two-thirds of these patients tend to experience relapse even after successful treatment by surgical resection. ${ }^{3}$ Many of these cases may develop metastases within two years after the resection of the primary tumour. Early diagnosis of colorectal cancer is associated with a good prognosis with a 5-year relative survival rate of $91 \%$ for localized cancer and $70 \%$ for cancer with regional invasion. 4 Thus patient prognoses will be gravely affected by the lack of methods for early detection of this cancer.

In recent years, the focus of research is to uncover the biological mechanisms underlying the pathogenesis and progression of colorectal cancer. Molecular alterations play a crucial role in the advancement and invasiveness of colorectal carcinoma. ${ }^{5}$ One of the events driving the cellular events is the epithelial to mesenchymal transition resulting in loss of cell-cell contact and increased motility of the cells. This change in phenotype enables the tumour cell to invade the surrounding tissues and also leads to metastasis to distant sites. Several molecules and signalling pathways are known to be involved in the cellular transition, thus contributing to the progression of colorectal cancer.6,7

Several biomarkers have been identified as indicators of epithelial-mesenchymal transition including E-cadherin, vimentin, and N-cadherin.8,9 Study of these molecular factors helps in the identification of subgroups with upregulation or downregulation of certain markers, indicating the need for a customized therapeutic approach for treatment. k. Decreased levels of E-cadherin expression were reported in many malignant cancers. ${ }^{10,11}$ In some of the cancer types, reduced E-cadherin expression was associated with loss of differentiation and correlated with an elevated risk of metastasis, indicating the importance of this glycoprotein in tumour invasion and metastasis. ${ }^{12,13}$ E-cadherin deregulation is involved in invasion, angiogenesis, as well as progression in many cancers including colorectal cancers. This cadherin is expected to be a useful marker of poor differentiation and invasiveness of colorectal cancer.

India has a low reported incidence of this cancer - 4.4 and 4.1 per 100000 cases, respectively in colon cancer and rectal cancer in men. ${ }^{14}$ But India has younger patients, and more patients present with advanced stage of colorectal cancer. ${ }^{15}$ In one of the reports, around $28 \%$ of the patients had metastases with the liver as the most common site of metastasis. ${ }^{15}$ The current system of TNM staging in colorectal cancer and clinicopathological features seems to be insufficient to identify the different cases with poor prognoses. Many of these cases show a different pattern of progression with reduced survival rates. There are very few studies evaluating the clinical profile of colorectal cancer in Indian patients. The majority of the studies are based on tumour and cell lines of Caucasian patients and thus most of the clinical management strategies are based on this population which may not be optimal for Indian patients with colorectal cancer. Moreover, we still do not have a reliable criterion for predicting aggressiveness to formulate strategies to intervene in metastases of colorectal cancer.

\section{Objectives}

1. To assess the pattern of expression of E-cadherin in colorectal cancer patients from a medical college in Kerala.

2. To determine the association between expression of Ecadherin and different morphological parameters as well as grade and stage of the tumour.

\section{METHODS}

In this cross-sectional study, a total of 43 specimens of histologically diagnosed colorectal cancers were received in the Department of Pathology of Government Medical College, Thrissur. The study was conducted for two years from January 2019 to February 2021. All these specimens received were included in the study.

Patients who were under treatment or had already taken treatment for colorectal cancer were excluded from the study. The sample size suggested for the study was 40 . Approval for the study was obtained from Institute Ethics Review Board.

Sections were taken for H\&E to assess the Grade and TNM stage and Immunohistochemical (IHC) staining of formalinfixed paraffin-embedded tissue was done. Sections of tissue were cut consecutively at 4-micrometre thickness. Tissue microarray was used for the analysis of IHC staining. Sections were deparaffinized and dehydrated. Antigen retrieval was performed by pressure cooking and stained with an Ecadherin IHC marker. E-cadherin staining was evaluated using a regular light microscope at the magnification of 40x.

\section{The Intensity of E-cadherin Staining in Tumour Cell was Scored as}

1. ECD++ if equal to or approaching that of normal epithelium.

2. ECD+ in cases where the staining was recognizable but weaker than normal epithelium.

3. ECD- expression was lost completely.

\section{Membranous Staining was Evaluated. For Cell Membrane Staining, Four Categories Were Used,}

0 ) No expression, no detectable staining in $<10 \%$ of the membranes

1) Weak but detectable discontinuous staining present in $10-$ $39 \%$ of the membranes

2) Moderate, clearly positive discontinuous staining present in $40-90 \%$ of the membranes and

3) Intense continuous staining of the membranes creates a honeycomb pattern. E-cadherin membrane index (I) was calculated with both the intensity of staining and fraction of positively stained cells. 


\section{Statistical Analysis}

Data thus obtained was entered in Microsoft office excel 2010 sheet. This was then analysed using software SPSS version 16.0. Frequency tables of different variables were analysed using the chi-square test. The findings are presented in appropriate charts and tables.

\section{RESULTS}

In this study, the majority of the subjects were in the age group of 51 - 70 years. The mean age of the participants was 58 years. Around $51 \%(\mathrm{~N}=22)$ were females and the rest $(\mathrm{N}=21)$ were males. Considering the tumour location, 20 cases $(46.4 \%)$ of the total 43 involved proximal colon, 13 cases (30.2\%) involved distal colon and the rest $(\mathrm{N}=10,23.3$ $\%$ ) involved rectum. The size of the tumour ranged from $1.5-$ $11 \mathrm{~cm}$. The majority of the tumours were $<5 \mathrm{~cm}$ in size. Details of the tumour are presented in Table 1.

\begin{tabular}{|c|c|c|c|}
\hline & Characteristics & Frequency & Percentage \\
\hline \multirow{3}{*}{ Tumor Location } & Proximal colon & 20 & 46.5 \\
\hline & Distal colon & 13 & 30.2 \\
\hline & Rectum & 10 & 23.3 \\
\hline \multirow{3}{*}{ Tumour Size } & $\leq 5 \mathrm{~cm}$ & 22 & 51.1 \\
\hline & $6-10 \mathrm{~cm}$ & 20 & 46.5 \\
\hline & $>10 \mathrm{~cm}$ & 1 & 2.3 \\
\hline \multirow{4}{*}{ Tumour Stage } & T1 & 1 & 2.32 \\
\hline & T2 & 9 & 20.93 \\
\hline & T3 & 30 & 69.76 \\
\hline & $\mathrm{T} 4$ & 3 & 6.97 \\
\hline \multirow{3}{*}{$\begin{array}{l}\text { Lymphatic Node } \\
\text { Status }\end{array}$} & N0 & 25 & 58.1 \\
\hline & N1 & 10 & 23.3 \\
\hline & $\mathrm{N} 2$ & 8 & 18.6 \\
\hline \multirow{3}{*}{ M Status } & M0 & 9.3 & 4 \\
\hline & M1 & 2.3 & 1 \\
\hline & $\mathrm{Mx}$ & 88.4 & 38 \\
\hline \multirow{4}{*}{$\begin{array}{c}\text { Tumour } \\
\text { Differentiation }\end{array}$} & Well & 33 & 76.7 \\
\hline & Moderate & 5 & 11.6 \\
\hline & Poorly & 2 & 4.7 \\
\hline & Mucinous & 3 & 7 \\
\hline \multirow{2}{*}{ Tumour Grade } & Low grade & 41 & 95.3 \\
\hline & High grade & 2 & 4.7 \\
\hline \multirow{5}{*}{ Staining pattern } & heterogeneous & 19 & 44.2 \\
\hline & homogenous & 24 & 55.8 \\
\hline & membranous & 7 & 16.3 \\
\hline & Membranous+cytoplasmic & 28 & 65.1 \\
\hline & cytoplasmic & 8 & 18.6 \\
\hline
\end{tabular}

A total of $69.76 \%(\mathrm{~N}=30)$ participants had stage 3 tumour, another $20.93 \%(\mathrm{~N}=9)$ had stage 2 tumour. The majority of the patients $(58.1 \%, \mathrm{~N}=25)$ did not have any lymph node metastasis (N0), while 10 (23.3\%) of them were in N1 lymph node status and 8 (18.6 \%) had N2 lymph node status. Metastasis could not be assessed in the majority of patients. Around 38 (88.4 \%) had Mx status, while one patient had M1 status and four of the subjects had M0 status. Tumour was well-differentiated in the majority of the participants $(\mathrm{N}=33)$, while five of the patients had a moderately differentiated tumour. Three of the participants (7\%) had a mucinous tumour. The low-grade tumour was recorded in $95.3 \%$ (41) participants while only two (4.7\%) had a high-grade tumour.

Both homogenous $(55.8 \%)$ and heterogeneous staining $(44.2 \%)$ were noted in the E-cadherin staining pattern among the participants. In this, more than half of the tumours $(65.1 \%)$ showed membranous + cytoplasmic staining, while the rest were distributed between membranous (16.3\%) and cytoplasmic (18.6\%) patterns of staining. A high E-cadherin index $(>2)$ was reported from more than $50 \%$ of the patients
$(\mathrm{N}=22)$, while the rest (48.8\%) had an E-cadherin index under 2. Fifteen participants with a high E-cadherin index showed a homogenous pattern of expression, while in patients with a low E-cadherin index, 12 showed a heterogeneous pattern of expression (Fig 2).

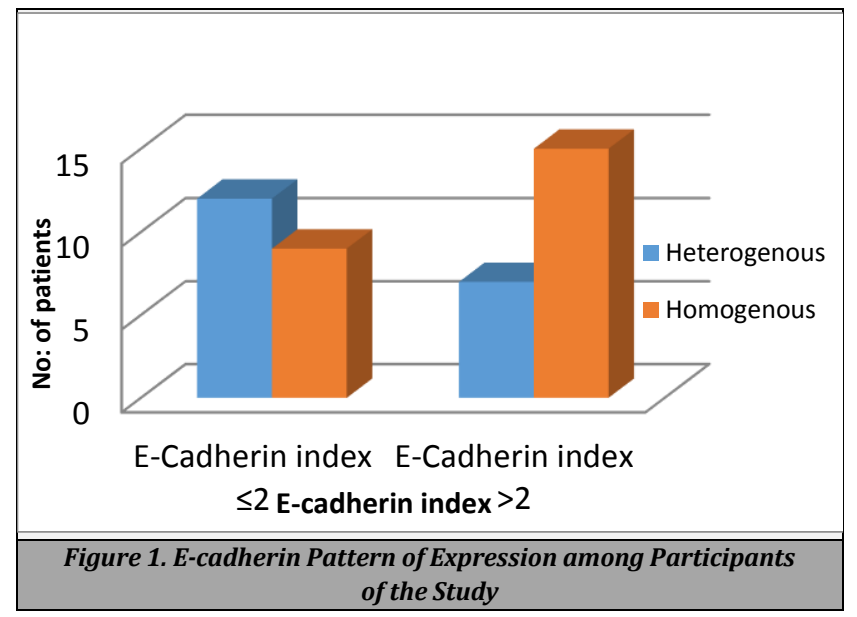

E-cadherin expression was not statistically associated with tumour differentiation in the patients (Table 2).

\begin{tabular}{|c|c|c|c|}
\hline & Characteristic & $\begin{array}{c}\text { E-Cadherin } \\
\text { Index } \leq 2(N)\end{array}$ & $\begin{array}{c}\text { E-Cadherin } \\
\text { Index }>2(N)\end{array}$ \\
\hline \multirow{2}{*}{$\begin{array}{l}\text { Pattern of } \\
\text { Expression }\end{array}$} & Heterogeneous & 12 & 7 \\
\hline & Homogenous & 9 & 15 \\
\hline \multirow{4}{*}{$\begin{array}{c}\text { Tumour } \\
\text { Differentiation }\end{array}$} & Well & 15 & 18 \\
\hline & Moderate & 4 & 1 \\
\hline & Poorly & 2 & 0 \\
\hline & Mucinous & 0 & 3 \\
\hline \multirow{3}{*}{$\begin{array}{c}\text { Histological } \\
\text { Grade of Tumour }\end{array}$} & Low Grade & 19 & 22 \\
\hline & High Grade & 2 & 0 \\
\hline & $\leq 5 \mathrm{~cm}$ & 10 & 12 \\
\hline \multirow[t]{2}{*}{ Size of Tumour } & $\overline{6-10 \mathrm{~cm}}$ & 10 & 10 \\
\hline & $>10 \mathrm{~cm}$ & 1 & 0 \\
\hline \multirow{3}{*}{ Tumour Location } & Proximal colon & 10 & 10 \\
\hline & Distal colon & 6 & 7 \\
\hline & Rectum & 5 & 5 \\
\hline \multirow{4}{*}{ Tumour Stage } & $\mathrm{T} 1$ & 0 & 1 \\
\hline & $\mathrm{T} 2$ & 3 & 6 \\
\hline & T3 & 16 & 14 \\
\hline & $\mathrm{T} 4$ & 2 & 1 \\
\hline \multirow{3}{*}{$\begin{array}{l}\text { Lymphatic Node } \\
\text { Status }\end{array}$} & No & 11 & 14 \\
\hline & N1 & 7 & 3 \\
\hline & $\mathrm{N} 2$ & 3 & 5 \\
\hline
\end{tabular}

Most of the low-grade tumours showed predominantly high E-cadherin index, but high-grade tumours consistently showed low E-cadherin index. Comparison of the size of the tumour with that of the E-cadherin index showed that the majority of the high E-cadherin index patients had a tumour size $\leq 5$. The majority of the patients had proximal colon as the location of the tumour, irrespective of the E-cadherin index. Seven of the patients in the high E-cadherin index group and six participants in the low E-cadherin index group had a tumour in the distal colon. Rectum was the site of a tumour in 5 participants each of high and low E-cadherin index group.

Comparing the T stage with that of the E-cadherin index, the majority $(\mathrm{N}=16)$ of the participants with low index were in stage 3. Fourteen participants with a high index of Ecadherin had stage 3 tumours. No statistically significant association was found between the $\mathrm{T}$ stage and E-cadherin index among the patients. The majority of the participants did not show any lymph node metastasis, and when present it was mostly in the low E-cadherin index group. Lymph node 
status also was not significantly associated with the Ecadherin index in this study.

The majority of the low-grade tumours $(\mathrm{N}=27)$ showed membranous + cytoplasmic staining in all low-grade tumours. Eight of the low-grade tumours showed aberrant cytoplasmic staining (Fig 1 -2). Normal staining was noted only in 6 cases of low-grade tumours and one case of the high-grade tumour.
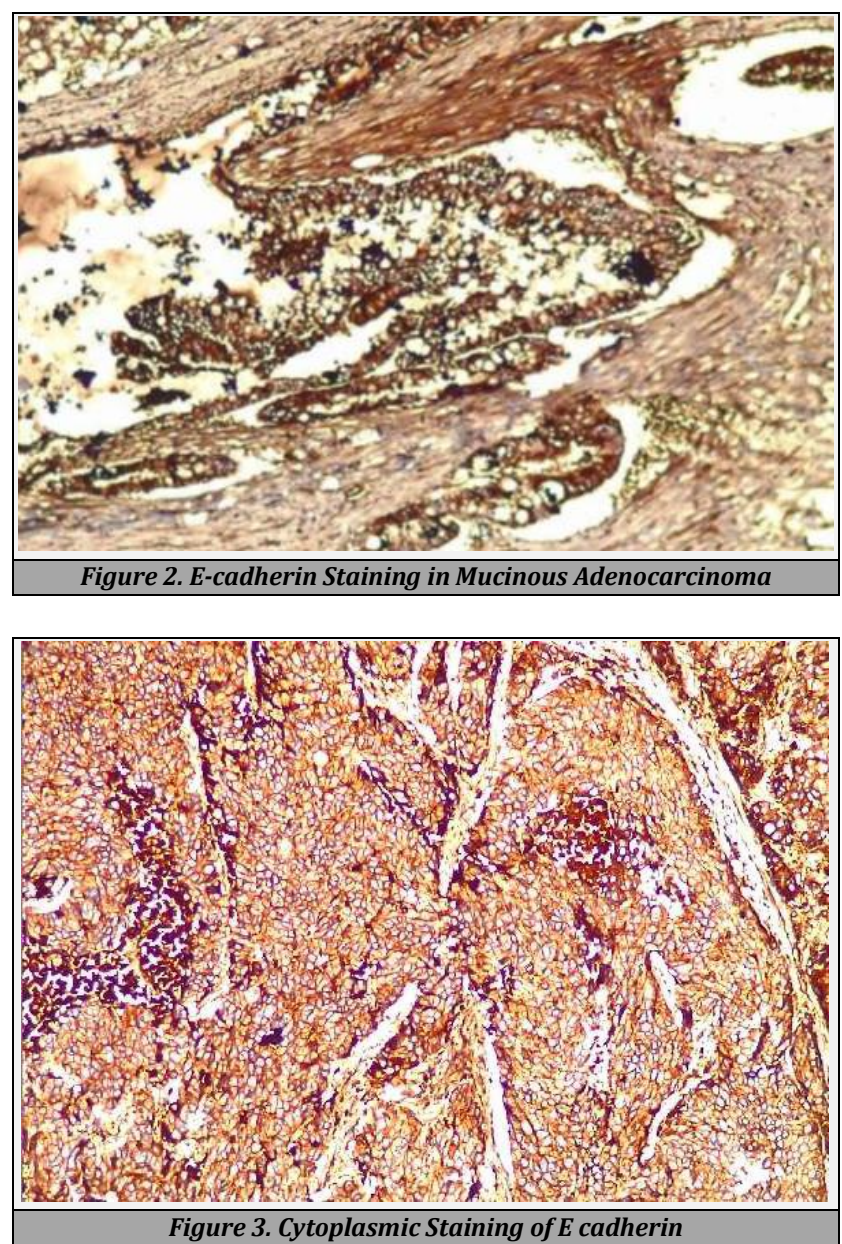

\section{DISCUSSION}

Colorectal cancer is a common cancer worldwide with a majority of cases reported from western countries. ${ }^{1}$ In India, despite the absence of strict population-based screening programs, the incidence rates have shown an increase in the last decade probably because of changing lifestyles and urbanization. The present study evaluated the expression of E-cadherin and its relation with clinicopathological features in colorectal carcinoma. Females $(\mathrm{N}=22)$ slightly outnumbered males $(\mathrm{N}=21)$ in this study.

E-cadherin belong to a large family of membraneassociated glycoproteins, mediating cell to cell adhesion playing a major role in tissue morphogenesis. ${ }^{16}$ This protein maintains the cohesiveness and epithelial integrity of colorectal cancers. ${ }^{17}$ Downregulation of E-cadherin is one of the key events leading to epithelial-mesenchymal transition, which is linked to invasiveness in several cancers. ${ }^{18-20}$ There seems to be a lack of consensus on the prevalence of $\mathrm{E}$ cadherin expression in colorectal cancers in literature with a 26-100\% range in E-cadherin positivity. ${ }^{21-23}$ E-cadherin expression is reported as higher in primary colorectal cancers in younger patients when compared to elderly people.

Epithelial cells of the colorectal region usually show strong membranous expression of E-cadherin at the borders, reflecting the localization of adhesion molecules. ${ }^{24}$ In the current study, a high E-cadherin index $(>2)$ was reported from more than $50 \%$ of the patients $(\mathrm{N}=22)$, while the rest had an E-cadherin index under 2. Concentration of E-cadherin was higher in patients with benign as well as with colorectal cancer as shown by Wilmanns et al. in a study conducted on 36 patients with colorectal cancers. ${ }^{25}$ Studies showed that the expression of E-cadherin was higher in the centre of the tumour when compared to that of the tumour margin. ${ }^{26}$

When we assessed the pattern of E-cadherin expression in the tumours, only $16 \%(\mathrm{~N}=7)$ showed pure membranous staining. Though not statistically associated with the histological grade or stage of the tumour, this indicates an aberrant staining pattern in colorectal carcinoma and is consistent with previous reports. ${ }^{27-28}$ E-cadherin membranous expression was found in more than half of the liver metastases in colorectal cancer and the authors commented that reiteration of membranous expression was important in metastases as the loss of expression on the membrane was just a transient process during which the cells detached from the primary tumour. ${ }^{24}$ Studies show that metastatic cells have increased E-cadherin expression when compared to primary colorectal cancer, suggesting the role of E-cadherin formation in neoplasms. In addition to being a crucial mechanism in the proliferation of cancer cells, a restatement of E-cadherin membranous expression may be an important step in the survival of the tumour cells. In the present study, more than $50 \%(\mathrm{~N}=24)$ of low-grade tumours showed homogenous staining comparable to benign tumours. Higher grade tumours showed heterogeneous staining with adjacent cells showing different patterns due to reduced expression of E-cadherin. Cytoplasmic or mixed expression was not noted in primary colorectal cancers in a study conducted by Bezdekova et al. as they found mostly membranous expression in the cells. ${ }^{29}$ Heterogeneous staining and local and distant progression of the tumour were significantly correlated in a study conducted by Bringuier et al. (1999) in bladder tumours. 30

Most of the studies showed a significant correlation between invasiveness and E-cadherin expression in other forms of cancers. ${ }^{28,31}$ An increased migration of colorectal cancer cells was found to be associated with E-cadherin expression in other studies. ${ }^{32}$ These studies indicate Ecadherin expression as a metastasis prediction marker and a prognostic marker in cancers including colorectal cancer.

But, the current study did not indicate any such association between invasive phenotype and E-cadherin expression which is in line with the study by Anna et al., where the invasion was not paralleled by loss of expression of E-cadherin. ${ }^{33}$ E-cadherin expression was considerably reduced in cases with lymph node metastases in this study. A statistically significant association was not present between lymph node metastases and expression of E-cadherin $(\mathrm{P}=0.296)$, though 25 out of 43 cases showed the presence of lymph node metastases in the present study. Palaghia et al. also reported the absence of correlation between E-cadherin 
expression and metastases to lymph nodes. ${ }^{28}$ The difference is shown in our study may be because of the change in qualitative expression of E-cadherin rather than quantitative changes, as the tumour cells continue to express the protein, albeit in an aberrant way. Cell lines from squamous cell carcinoma and gastric adenocarcinomas also did not show strong invasiveness with reduced E-cadherin expression. ${ }^{34,35}$ When the expression of E-cadherin was suppressed by genetic engineering techniques in colorectal cancer cell lines, the replicative ability, invasion capacity, and resistance to growth inhibitors of the cells increased considerably. ${ }^{36}$

E-cadherin expression seems to be preserved in well and moderately differentiated tumours, while poorly differentiated tumours showed a marked decrease in the expression of this protein. ${ }^{28}$ Progressive loss of differentiation was associated with loss of E-cadherin expression in the study by Anna et al. who also reported a gradual loss of E-cadherin from adenoma to high-grade carcinoma $^{33}$ E-cadherin expression plays a major role in maintaining a well-differentiated pattern of the glands, so much so that loss of its expression results in undifferentiated tumours and a greater risk of invasiveness. In our study, the decrease in E-cadherin expression was limited to the least differentiated specimens. This difference may be attributed to the differences in the methodologies employed for sample selection and immunohistochemical staining.

Though well studied, the existing data on E-cadherin downregulation is highly variable and its relevance to the progression of colorectal cancers is not fully demonstrated. A recent review of published studies on cadherin downregulation shows that more than 100 cadherins are downregulated in colorectal cancer. ${ }^{37}$ Co-expression pattern analysis of $\alpha$-catenin and E-cadherin in colorectal adenocarcinoma shows that invasiveness of tumour is associated with downregulation of $\alpha$-catenin regardless of Ecadherin expression. ${ }^{38}$ E-cadherin vascular endothelial growth factor-A (VEGFA) combination was reported as a prognostic marker in metastatic colorectal cancer. In this study, only $29 \%$ of the cases showed reduced E-cadherin expression and were not statistically correlated with invasiveness or metastasis. While in sporadic colorectal neoplasms, nuclear localization of $\beta$-catenins was found to be higher at the invasive margin and no consistent relationship was found between expression of E-cadherin and $\beta$-catenins in lymph node deposits. ${ }^{39}$ Thus expression of multiple cadherins should be studied to understand invasion and metastases in colorectal cancers. More large, functional studies are necessary to clarify the ability of these proteins to act as predictive biomarkers of tumour progression and invasion in colorectal cancer.

The genetic factors that determine the different subgroups in colorectal cancer were not considered which is one of the limitations of the study apart from a small sample size. Studying genetic markers that differentiate the types of tumours will help in developing a customized therapeutic approach for the treatment of the same. Further, the correlation between E-cadherin expression and survival rate was not an objective of the study, which would have helped in associating the marker with a prognosis of the disease.

\section{CONCLUSIONS}

There is a significant difference in E-cadherin expression between low and high grade colorectal cancer. High-grade tumours showed a more heterogeneous staining pattern correlating with increased invasiveness of the tumour. The heterogeneous pattern of staining in E-cadherin among adjacent cells of the tumour results from reduced expression of the protein in the tumour. No significant association exists between E-cadherin expression and other tumour features including grade differentiation, TNM staging and other morphological features like location and size of the tumour.

Data sharing statement provided by the authors is available with the full text of this article at jemds.com.

Financial or other competing interests: None.

Disclosure forms provided by the authors are available with the full text of this article at jemds.com.

\section{REFERENCES}

[1] Edwards MS, Chadda SD, Zhao Z, et al. A systematic review of treatment guidelines for metastatic colorectal cancer. Colorectal Dis 2012;14(2):e31-47.

[2] Arnold M, Sierra MS, Laversanne M, et al. Global patterns and trends in colorectal cancer incidence and mortality. Gut 2017;66(4):683-91.

[3] Geoghegan JG, Scheele J. Treatment of colorectal liver metastases. British Journal of Surgery 1999;86(2):15869.

[4] Institut National Du Cancer (INCA), corp-author Les traitements du cancer du côlon, collection Guides patients Cancer info. http://www.e-cancer.fr/Patientset-proches/Les-cancers/Cancer-du-colon/Points-cles INCA. 2010

[5] Fearon EF, Vogelstein B. A genetic model for colorectal tumorigenesis. Cell 1990;61(5):759-67.

[6] Chaffer CL, Juan BPS, Lim E, et al. EMT, cell plasticity and metastasis. Cancer Metastasis Rev 2016;35(4):645-54.

[7] Espinoza I, Miele L. Deadly crosstalk: notch signaling at the intersection of EMT and cancer stem cells. Cancer Lett 2013;341(1):41-5.

[8] Arias AM. Epithelial mesenchymal interactions in cancer and development. Cell 2001;105(4):425-31.

[9] He X, Chen Z, Jia M, et al. Downregulated E-cadherin expression indicates worse prognosis in Asian patients with colorectal cancer: evidence from meta-analysis. PLoS One 2013;8(7):e70858.

[10] Shiozaki H, Oka H, Inoue M, et al. Ecadherin mediated adhesion system in cancer cells. Cancer 1996;77(8 Suppl):1605-13.

[11] Lipponen P, Saarelainen E, Ji H, et al. Expression of Ecadherin (E-CD) as related to other prognostic factors and survival in breast cancer. J Pathol 1994;174(2):1019.

[12] Sanders DS, Perry I, Hardy R, et al. Aberrant P-cadherin expression is a feature of clonal expansion in the gastrointestinal tract associated with repair and neoplasia. J Pathol 2000;190(5):526-30. 
[13] Bendardaf R, Elzagheid A, Lamlum H, et al. E-cadherin, CD44s and CD44v6 correlate with tumour differentiation in colorectal cancer. Oncol Rep 2005;13(5):831-5.

[14] Consensus Document for management of colorectal cancer-prepared as an outcome of ICMR Subcommittee on colorectal cancer. Indian Council of Medical Research 2014.

[15] Patil PS, Saklani A, Gambhire P, et al. Colorectal cancer in India: an audit from a tertiary center in a low prevalence area. Indian J Surg Oncol 2017;8(4):484-90.

[16] Tsanou E, Peschos D, Batistatou A, et al. The E-cadherin adhesion molecule and colorectal cancer. A global literature approach. Anticancer Res 2008;28(6):3815-26.

[17] Takeichi M. Cadherin cell adhesion receptors as a morphogenetic regulator. Science 1991;251(5000):1451-5.

[18] Thiery JP. Epithelial-mesenchymal transitions in tumour progression. Nat Rev Cancer 2002;2(6):442-54.

[19] Nagathihalli NS, Merchant NB. Src-mediated regulation of E-cadherin and EMT in pancreatic cancer. Front Biosci 2012;17:2059-69.

[20] Cheng JC, Auersperg N, Leung PCK. EGF-induced EMT and invasiveness in serous borderline ovarian tumor cells: a possible step in the transition to low-grade serous carcinoma cells? Plos One 2012;7(3):e34071.

[21] Senol S, Ceyran AB, Kosemetin D, et al. Immunohistochemical profile of tumor pathways and prognostic significance in colon adenocarcinomas. J Environ Pathol Toxicol Oncol 2017;36(1):29-41.

[22] Sayar I, Akbas EM, Isik A et al. Relationship among mismatch repair deficiency, CDX2 loss, p53 and Ecadherin in colon carcinoma and suitability of using a double panel of mismatch repair proteins by immunohistochemistry. Pol J Pathol 2015;66(3):246-53.

[23] Bondi J, Bukholm G, Nesland JM, et al. An increase in the number of adhesion proteins with altered expression is associated with an increased risk of cancer death for colon carcinoma patients. Int J Color Dis 2006;21(3):231-7.

[24] Gagliardi G, Kandemir O, Liu D, et al. Changes in Ecadherin immunoreactivity in the adenoma-carcinoma sequence of the large bowel. Virchows Arch 1995;426(2):149-54.

[25] Wilmanns C, Grossmann J, Steinhauer S, et al. Soluble serum E-cadherin as a marker of tumour progression in colorectal cancer patients. Clin Exp Metastasis 2004;21(1):75-8.

[26] Kim JC, Roh SA, Kim HC, et al. Coexpression of carcinoembryonic antigen and E-cadherin in colorectal adenocarcinoma with liver metastasis. J Gastrointest Surg. 2003;7(7):931-8.

[27] Khoursheed MA, Mathew TC, Makar RR, et al. Expression of E-cadherin in human colorectal cancer. Surgeon 2003;1(2):86-91.

[28] Palaghia M, Mihai C, Lozneanu L, et al. E-cadherin expression in primary colorectal cancer and metastatic lymph nodes. Rom J Morphol Embryol 2016;57(1):205-9.

[29] Bezdekova M, Brychtova S, Sedlakova E, et al. Analysis of Snail-1, E-cadherin and claudin-1 expression in colorectal adenomas and carcinomas. Int J Mol Sci 2012;13(2):1632-43.

[30] Bringuier PP, Giroldi LA, Umbas R, et al. Mechanisms associated with abnormal E-cadherin immunoreactivity in human bladder tumors. Int J Cancer 1999;83(5):591-5.

[31] Okugawa $Y$, Toiyama $Y$, Inoue $Y$, et al. Clinical significance of serum soluble E-cadherin in colorectal carcinoma. J Surg Res 2012;175(2):e67-73.

[32] Masur K, Lang K, Niggemann B, et al. High PKC alpha and low E-cadherin expression contribute to high migratory activity of colon carcinoma cells. Mol Biol Cell 2001;12(7):1973-82.

[33] van der Wurff AA, Vermeulen SJ, van der Linden EP, et al. Patterns of $\alpha$ - and $\beta$-catenin and E-cadherin expression in colorectal adenomas and carcinomas. J Pathol 1997;182(3):325-30.

[34] Kinsella AR, Bowie GL, Field JK, et al. Expression of the cell-cell adhesion molecule E-cadherin in tongue carcinoma cell lines. J Laryngol Otol 1994;108(11):95761.

[35] Shimoyama Y, Hirohashi S. Expression of E- and Pcadherin in gastric carcinomas. Cancer Res 1991;51(8):2185-92.

[36] Chen X, Wang Y, Xia H, et al. Loss of E-cadherin promotes the growth, invasion and drug resistance of colorectal cancer cells and is associated with liver metastasis. Mol Biol Rep 2012;39(6):6707-14.

[37] Losi L, Zanocco-Marani T, Grande A. Cadherins downregulation: towards a better understanding of their relevance in colorectal cancer. Histol Histopathol 2020;35(12):1391-402.

[38] Gofuku J, Shiozaki H, Tsujinaka T, et al. Expression of Ecadherin and alpha-catenin in patients with colorectal carcinoma. Correlation with cancer invasion and metastasis. Am J Clin Pathol 1999;111(1):29-37.

[39] El-Bahrawy MA, Poulsom R, Jeffery R, et al. The expression of E-cadherin and catenins in sporadic colorectal carcinoma. Hum Pathol 2001;32(11):1216-24. 